\title{
An Update on Isolation of Extensively Drug Resistant (XDR) Salmonella enterica from Blood Cultures in a Tertiary Care Centre
}

\author{
Sadia Hameed, Mateen Izhar, Anwaar Basheer, Chetan Lal, Shazia Rishi, Abdul Basit \\ Department of Microbiology, Shaikh Zayed Medical Complex, Lahore
}

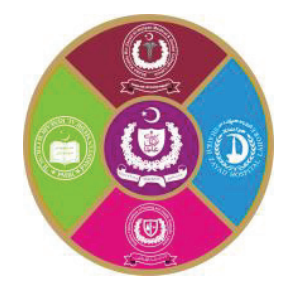

\begin{abstract}
Introduction: Typhoid fever is a public health issue, burdening many regions of the world with poor socioeconomic background. Management of this disease faces the major hurdle of antimicrobial resistance. The present study reveals current pattern of antimicrobial susceptibility among Salmonella enterica (Salmonella typhi \& Salmonella paratyphi A) blood culture isolates from typhoid fever cases. Regular data collection about Salmonella infections and their response to antimicrobial agents, coupled with a long term commitment to providing adequate health information systems, is the key to effective planning and policy formation against typhoid fever. Aims \& Objectives: To evaluate the prevalence of ceftriaxone resistant Salmonella enterica isolates from blood cultures in Shaikh Zayed Medical Complex for updating nosocomial antimicrobial resistance data. Place and duration of study: This research study was conducted at Shaikh Zayed Medical Complex, Lahore from March 2018 to May 2019. Material \& Methods: Blood cultures positive for Salmonella enterica were analyzed, taking into consideration the gender \& age of patients with typhoid fever. Antimicrobial susceptibility testing was carried out through disc diffusion method. The recommended antimicrobial agents for S. typhi (Salmonella typhi) \& S. paratyphi A (Salmonella Parathyphi A) (as per CLSI, USA 2018/2019 guidelines; described later), were tested and analyzed during this study with the main focus on $3^{\text {rd }}$ generation cephalosporin resistance pattern. Among second line treatment options, meropenem and azithromycin were selected for study. Results: Out of 899 isolates of Salmonella enterica (from 13964 samples for blood culture), 849 (94.4\%) were S. typhi and 50 (5.6\%) were S. paratyphi A. Of these, $57.1 \%$ isolates were from males, $42.9 \%$ from females, $81.7 \%$ from children (age less than 12 years) and $18.3 \%$ were from adults (age more than 12 years). A continuously rising resistance percentage was observed for $3^{\text {rd }}$ generation cephalosporins over the span of fifteen months. It was $43.4 \%$ for ceftriaxone (CRO). No case of meropenem and azithromycin resistance was observed during study period. Conclusion: Demographic information was provided by this study regarding high level of resistance among Salmonella enterica isolates particularly $S$. typhi .The first line antibiotic drugs were a complete failure in ongoing outbreak of typhoid fever. The novel results of this study are the high resistance percentages for $3^{\text {rd }}$ generation cehalosporins, whether oral or parenteral. This result is worrisome as it will leave us with no option but to resort to second line drugs. However, an increasing trend of blood culturing was observed in this research. Extensively drug resistant (XDR) Salmonella infection has definitely highlighted the importance of blood culture and its use as a more preferred diagnostic tool.
\end{abstract}

Key words: Typhoid fever, Extensively drug resistant (XDR) Salmonella enterica, (S. typhi \& S. paratyphi A), Antimicrobial Resistance, CRO (Ceftriaxone) resistance.

\section{INTRODUCTION}

$S_{\text {ix major disease outbreaks have been reported in }}$ Pakistan in year 2018: Extensively drug resistant (XDR) typhoid fever, chicken pox, dengue fever, poliomyelitis, chikungunya and crimean congo haemorrhagic fever $(\mathrm{CCHF})^{1}$. Typhoid fever is a systemic febrile illness requiring urgent antibiotic therapy. Approximately 12-27 million typhoid fever cases occur annually, worldwide ${ }^{2}$. In 2014 , out of
0.2 million deaths associated with typhoid fever, more than $90 \%$ occurred in Asia ${ }^{3}$.

Moreover, Pakistan and India account for a very high incidence of typhoid fever compared to other South Asian countries such as China, Indonesia and Vietnam $^{4}$. The mortality rate rises up to $30 \%$ resulting from inappropriate treatment (as serious life threatening complications such as small bowel perforation and meningitis can occur), whereas it is reduced up to $0.5 \%$ with proper remedy ${ }^{5,6}$. 
S. typhi accounts for a major count of typhoid fever cases. The relative contribution and incidence of Salmonella paratyphi A, a less severe disease causing agent than $S$. typhi, is not well understood, as most studies in Indian Subcontinent have mainly focused on S. typhi. However a rise in S. paratyphi A infection has been reported ${ }^{7}$.

Historically, the first line treatment options for typhoid fever have been chloramphenicol, ampicillin, and cotrimoxazole ${ }^{3}$. In early 1970s, resistance to chloramphenicol emerged in Salmonella enterica strains followed by resistance to ampicillin and cotrimoxazole. Since 1990s, many epidemics of typhoid fever occurred due to multidrug resistant Salmonella typhi (MDR-ST). This led to the use of flouroquinolones as first line antibiotic therapy options for typhoid fever, worldwide. But then, there emerged quinolone resistant strains with decreased susceptibility to ciprofloxacin ${ }^{8}$. Over the past two decades, global spread of a dominant, usually MDR, haplotype of $S$. typhi called H58 (genotype 4.3.1) has been observed. It emerged in early 1990s, has ability to maintain and traffic MDR plasmids and is associated with resistance to fluoroquinolones through common gyrA/parC mutations. It is prevalent across Southeast Asia and parts of Africa and Oceania. Global dispersal of this lineage is now a real possibility ${ }^{9}$.

In Pakistan, MDR and quinolone resistant $\mathrm{S}$. enterica strains are of great concern for public health. As a result of emergence and spread of quinolone resistant $S$. typhi in Pakistan, $3^{\text {rd }}$ generation cephalosporins such as oral cefixime or parenteral ceftriaxone became empirical treatment options $^{10}$.

Since November 2016, a large proportion of ceftriaxone resistant cases have been reported in Sindh particularly Hyderabad and Karachi and these strains were XDR (extensively drug resistant) i.e. non susceptible to chloramphenicol, ampicillin, cotrimoxazole, flouroquinolones and $3^{\text {rd }}$ generation cehalosporins ${ }^{11}$.

As continuous monitoring and evaluation of local antimicrobial susceptibility testing (AST) profiles are in practice to update therapeutic guidelines, the present study was done in a tertiary care hospital in Lahore, keeping in view XDR Salmonella enterica outbreak.

\section{MATERIAL AND METHODS}

In this prospective Study Salmonella enterica ( $S$. typhi \& $S$. paratyphi A) isolates were retrieved from blood culture samples received at Microbiology Laboratory from different departments of Shaikh
Zayed Medical Complex, Lahore during March 2018 - May 2019. A sum total of 13964 blood samples were processed for culture during study period. Data related to age, gender, serotypes of isolates and AST profiles was collected .Blood samples were first cultured in Tryptic Soy Broth followed by subculture on Mac Conkey and Blood agar under optimal conditions. Positive blood cultures were subjected to Identification (ID) and AST profiles using biochemical panel (API 20E) and latest CLSI recommendations, respectively. Isolates identified as Salmonellae were manually confirmed by slide agglutination test with polyvalent $\mathrm{O}$ antiserum and serovar specific antisera.

CLSI suggests following drugs to be reported for blood culture isolates of Salmonella enterica: ampicillin, fluoroquinolone, trimethoprimsulfamethoxazole (cotrimoxazole), $3^{\text {rd }}$ generation cephalosporin, chloramphenicol (if requested) and azithromycin. In our study, the evaluation of antibiogram was carried out as per CLSI. Azithromycin and meropenem (carbapenem) were taken into consideration as second line drugs.

\section{Statistical analysis:}

The data was computed using MS Excel and results were calculated and presented as column or row percentages.

\section{RESULTS}

Out of 13964 processed blood samples, 899 Salmonella enterica positive blood cultures were isolated over the span of 15 months. of these, 849 (94.4\%) were identified as $S$. typhi and $50(5.6 \%)$ as S. paratyphi A. (2 isolates identified as Salmonella paratyphi B were excluded from study due to low count). The age of the patients suffering from typhoid fever was from 2 months -67 years. Pediatric patients $81.7 \%$ accounted for majority of cases while adults were $18.3 \%$. Age distribution is shown in Fig-1 and gender distribution in Table-1.

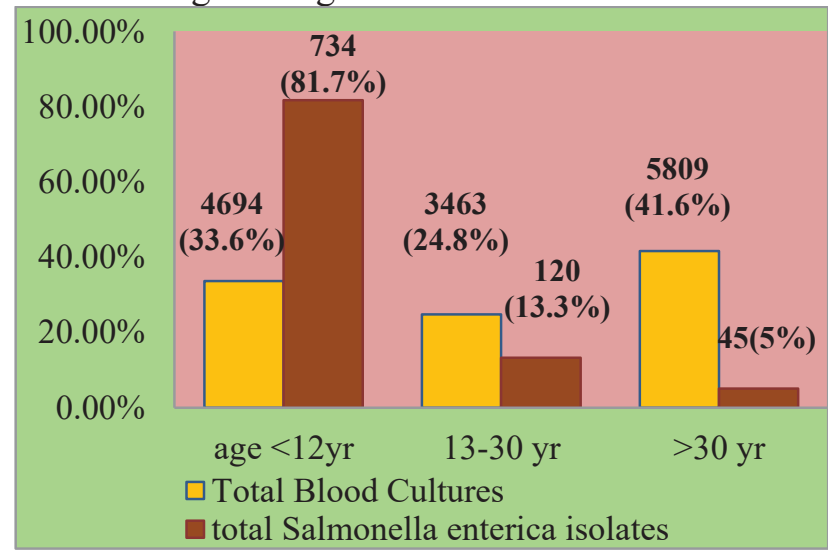

Fig-1: Age distribution 


\begin{tabular}{|l|c|c|c|}
\hline & $\begin{array}{c}\text { Total } \\
\text { Males }\end{array}$ & $\begin{array}{c}\text { Total } \\
\text { Females }\end{array}$ & Total \\
\hline Total Blood Cultures & $\begin{array}{c}8923 \\
(63.9 \%)\end{array}$ & $\begin{array}{c}5041 \\
(36.1 \%)\end{array}$ & 13964 \\
\hline $\begin{array}{l}\text { Total Salmonella } \\
\text { enterica } \text { isolates }\end{array}$ & $\begin{array}{c}513 \\
(57.1 \%)\end{array}$ & $\begin{array}{c}386 \\
(42.9 \%)\end{array}$ & 899 \\
\hline $\begin{array}{l}\text { Total CRO-Resistant } \\
\text { S.enterica isolates }\end{array}$ & $\begin{array}{c}182 \\
(35.4 \%)\end{array}$ & $\begin{array}{c}196 \\
(50.8 \%)\end{array}$ & 390 \\
\hline
\end{tabular}

Table-1: Gender distribution

Out of $43.4 \%$ CRO-Resistant strains of XDR $S$. enterica, percentage was $44.2 \%$ and $17.6 \%$ for $S$. typhi and $S$. paratyphi A respectively. S. enterica isolates were $42.1 \%$ resistant to cefixime. Out of this, $43.7 \%$ resistance was observed in S. typhi and $11.8 \%$ in S. paratyphi A. Following is a graph showing total number of blood cultures received in each month of research study (Fig-2).

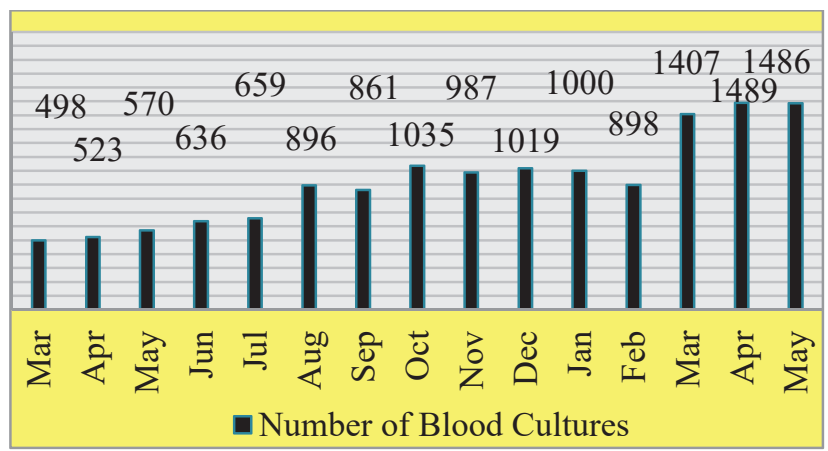

Fig-2: Number of Blood Cultures (March 2018-May 2019)

There is 2.8 times increase in the number of total blood cultures, over a span of 15 months. In addition, when three months (March, April and May) of two consecutive years (2018 \& 2019) were compared, there was a significant increase of $275 \%$ in the count.

A graph showing consistently rising resistance percentage to $3^{\text {rd }}$ generation cephalosporins i.e. ceftriaxone (CRO) among $\mathrm{S}$. enterica isolates over the span of 15 months is shown in Fig-3. It shows ever increasing percentages, even per month of the ongoing outbreak.

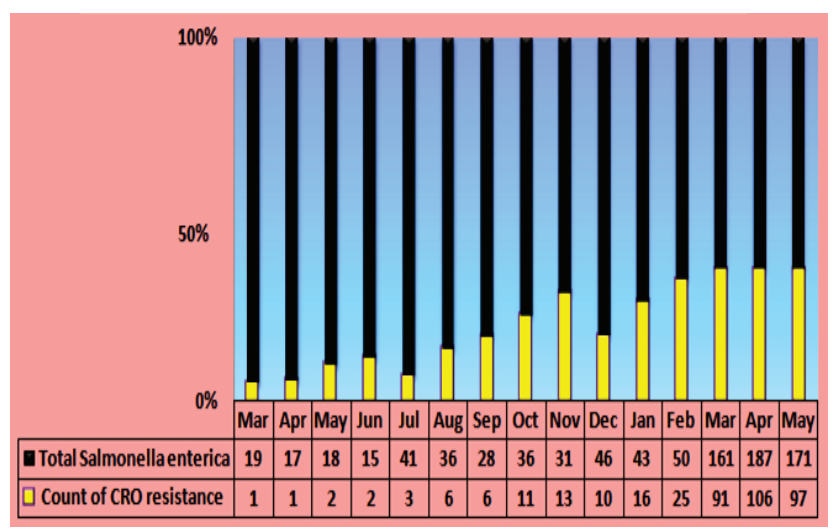

Fig-3: CRO-resistant Salmonella enterica isolates vs total Salmonella enterica isolates (March 2018 - May 2019)

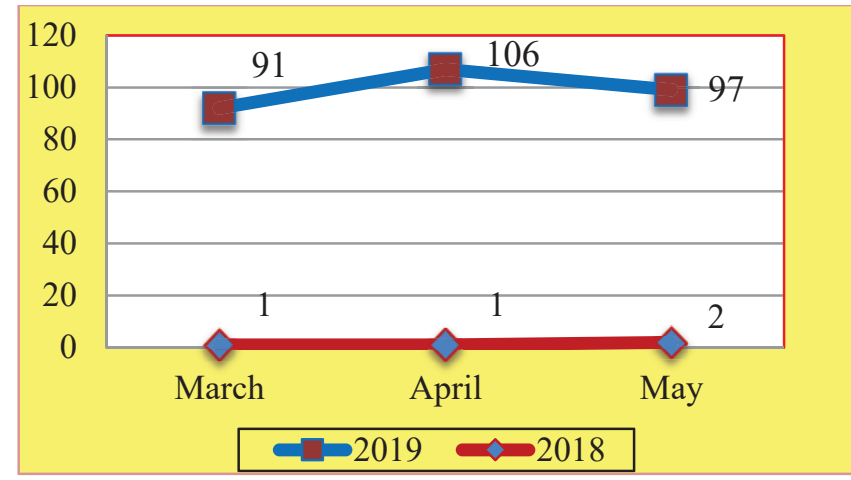

Fig-4: Comparison of CRO - Resistance (2018 vs 2019)

Fig-4 shows comparison of $3^{\text {rd }}$ generation cephalosporin (ceftriaxone) resistance for three months (March, April and May) of two consecutive years 2018 and 2019. There was a marked difference in the resistance pattern of the antimicrobial agent when compared after one year. Moreover, all isolates tested for azithromycin and meropenem were susceptible to these antibiotics.

\section{DISCUSSION}

Pakistan is currently facing the largest outbreak of extensively drug resistant invasive Salmonellosis. Reports from various districts have confirmed this fact $^{1}$.

A study at Hyderabad revealed largest outbreak of CRO-resistant S. typhi in the history of Pakistan that started at November 2016. Investigation discovered the likely reason of fecal contamination of water supply, contact with infected individuals and irrational antibiotic use. ${ }^{11}$ Our present study was aimed to monitor the ever changing landscape of S.enterica antimicrobial resistance. $3^{\text {rd }}$ generation cephalosporin resistance of XDR S. typhi was the main focus. Parenteral forms of $3^{\text {rd }}$ generation cephalosporins, considered as empirical therapy options in complicated typhoid fever cases before this outbreak, have equivalent efficacy when used in typhoid fever complications (e.g. meningitis) whereas cefixime qualifies as highly effective and safe oral drug with an efficacy of $92.5 \%$ in a study. ${ }^{12,13}$ These drugs have been proved as resistant in almost half of the cases of typhoid fever in our study. The count of total blood cultures, $S$. enterica isolates and ceftriaxone resistant $S$. enterica isolates were low in earlier months but all these parameters increased towards the end of research study as depicted in Fig-2 \& 3. For investigation of enteric fever, increasing trend for blood culturing reinforced by recent infection control policies, has definitely highlighted its importance as gold standard diagnostic tool compared to different non 
significant serological tests. In this way, it has yielded increased count of CRO-resistant $S$. enterica strains as well. This high cephalosporin resistance has also been reported from other areas of Asia (i.e. Bangladesh and India). ${ }^{11}$ Several case reports from west have raised the same issue e.g. a Canadian report revealed first case of CRO-resistant $S$. typhi in a three year old child who visited Karachi, Pakistan and got sick. ${ }^{14}$ Many other serovars of $S$. enterica have also been implicated in cephalosporin resistance for example XDR S. enterica serovar Oranienburg originated in Pakistan in recent years ${ }^{15}$. Although maximum percentage of blood culture samples was retrieved from patients of more than 30 years of age in our research, the pediatric age group $(<12$ years) had invasive Salmonellosis at its peak $(81.7 \%)$, a result consistent with many other research studies. The likely reasons considered for such higher resistance percentage are earlier consultation for treatment by the guardians of children, low trend of self-medication (antibiotic use) and increased microbial load (i.e. colony forming units $/ \mathrm{ml}$ of blood) in children as compared to adults. Among adults, male preponderance (57.1\%) was observed for typhoid fever cases, a result consistent with many other research studies e.g. an Indian research showed 58\% male typhoid cases $^{16}$ and a study in Bangladesh revealed 54\% cases ${ }^{17}$ in contrast to an Eastern Anatolian research showing male to female ratio of $1: 1.5(61: 93){ }^{18}$ Social and behavioral variations in different areas of world /age group affected or both might be the reason for difference. ${ }^{11}$ However, XDR Salmonella infection cases were from female patients mainly in the current study.

All strains tested for azithromycin and meropenem were $100 \%$ susceptible to these agents, a result same as many other studies.

\section{CONCLUSION}

The in vitro susceptibility patterns of 899 Salmonella enterica (S. typhi \& S. paratyphi A) isolates showed consistently high resistance to $3^{\text {rd }}$ generation cephalosporins (parenteral as well as oral), the drugs deemed as empirical therapy options in recent past. For these typhoid fever causing XDR strains, $2^{\text {nd }}$ line agents like carbapenems and azithromycin seem to be the last resort drugs. That is really an alarming situation as it in turn may have serious implications in our resource poor country due to high cost and little accessibility.

\section{Acknowledgements:}

We owe special thanks to Prof. Khalid Mehmood (Microbiology Department, College of Medicine \& Dentistry University of Lahore) and Dr. Aqsa Aslam (Assistant Professor of Microbiology at Sharif Medical and Dental College, Lahore) for reviewing and editing the article.

\section{REFERENCES}

1. World Health Organization Regional Office for Eastern Mediterranean. Weekly epidemiological monitor: disease outbreaks in Eastern Mediterranean Region (EMR), January to December 2018. Cairo, Egypt: World Health Organization Regional Office for Eastern Mediterranean; 2018.

2. Mogasale V, Maskery B, Ochiai RL, et al. Burden of typhoid fever in low-income and middle income countries: a systematic literature based update with risk factor adjustment. Lancet Globe Health. 2014; 2:e570-80.

3. Crump JA, Luby SP, Mintz ED. The global burden of typhoid fever. Bull world Health Organ. 2004; 82:346-53.

4. Ochiai RL, Acosta CJ, Danovaro-Holliday MC et al. Domi Typhoid Study Group. A study of typhoid fever in five Asian countries: disease burden and implication for controls. Bull world Health Organ. 2008; 86: 260-68.

5. Cooke FJ, Wain J. The Emergence of Antibiotic Resistance in Typhoid Fever. Travel Med Infect Dis. 2004; 2: 67-74.

6. Parry C, Hien T, Dougan G, et al. Typhoid fever. N Engl J Med. 2002; 347: 1770-82.

7. Saxena SN, Sen R. Salmonella paratyphi A infection in India: incidence and phage types. Trans R Soc Trop Med Hyg. 1966; 60: 409-11.

8. Walia M, Gaind R, Mehta R, Paul P, Aggarwal $\mathrm{P}$, Kalaiwani M. Current perspectives of enteric fever: a hospital-based study from India: Ann Trop Paediatr. 2005; 25: 161-74.

9. Wong VK, Baker S, Pickard DJ, et al. Phylogeographical analysis of the dominant multidrug- resistant H58 clade of Salmonella Typhi identifies inter- and intracontinental transmission events. Nat Genet. 2015; 47:632-9.

10. Qamar FN, Azmatullah A, Kazi A, et al. A three year review of antimicrobial resistance of Salmonella enterica serovars Typhi and Paratyphi A in Pakistan. J Infect Dev Ctries. 2014; 8: 981-6.

11. Qamar FN, Yousafzai M, Khalid M, et al. Outbreak investigation of ceftriaxone-resistant Salmonella enterica serotype Typhi its risk 
factors among the general population in Hyderabad, Pakistan; a matched case-control study. The Lancet Infectious Diseases. 2018; 12: $1368-76$.

12. Kavoliotis J, Tsiaousi A, Papavasiliou D, Kansouzidou A. Non-typhoid Salmonella meningitis. Scandinavian journal of infectious diseases. 1994; 26.4: 403-5.

13. Chaudhry MK, Rayamajhi BS, Paudel K, et al. Efficacy of cefixime in the treatment of typhoid fever. International Journal of Pharmaceutical \& Biological Archives. 2014; 4(2): 307-9.

14. Wong W, Al Rawahi H, Patel S, Yau Y, Eshaghi A, et al. The first Canadian Pediatric case of extensively drug-resistant Salmonella Typhi originating from an outbreak in Pakistan and its implication for empiric antimicrobial choices. IDCases. 2019; 15:e00492.

15. Yang WC, Chan $\mathrm{OW}, \mathrm{Wu} \mathrm{TL}$, et al. Development of ceftriaxone resistance in Salmonella enterica serotype Oranienburg during therapy for bacteremia. Journal of Microbiology, Immunolgy and Infection. 2016; $1: 41-5$.

16. Capt RMS, Capt KBS, Col SML, Col AN. Outbreak of multidrug resistant S Typhi enteric fever in Mumbai Garrison. Med J Armed Forces India. 2005; 61:148-50.

17. Ram P, Naheed A, Brooks W, et al. Risk factors for typhoid fever in slum in Dhaka, Bangladesh. Epidemiol Infect. 2007; 135:458-65.

18. Aypak A, Çelik AK, Aypak C, Çikman Ö. Multidrug resistant typhoid fever outbreak in Ercek Village-Van, Eastern Anatolia, Turkey: clinical profiles, sensitivity patterns and response to antimicrobials. Trop Doct. 2010; 40: 160-2.

\section{The Authors:}

Dr. Sadia Hameed,

P.G. Trainee,

Department of Microbiology,

Shaikh Zayed Medical Complex, Lahore.

Prof. Mateen Izhar

Head of Microbiology Department,

Shaikh Zayed Medical Complex, Lahore.

Dr. Anwaar Basheer,

Associate Professor,

Department of Microbiology,

Shaikh Zayed Medical Complex, Lahore.

Dr. Chetan Lal,

Assistant Professor,

Department of Microbiology,

Shaikh Zayed Medical Complex, Lahore.

Dr. Shazia Rishi,

Senior Medical Officer,

Department of Microbiology,

Shaikh Zayed Medical Complex, Lahore.

Abdul Basit,

Laboratory Manager,

Department of Microbiology,

Shaikh Zayed Medical Complex, Lahore.

\section{Corresponding Author:}

Dr. Sadia Hameed,

P.G. Trainee,

Department of Microbiology,

Shaikh Zayed Medical Complex, Lahore.

E-mail: sadiahameed888@gmail.com 